\title{
The Absorptive Part of the Nucleus-Nucleus Potential in a Semiclassical Approach
}

\author{
A.H. Blin, M. Brack, B. Hiller, and E. Werner \\ Institut für Theoretische Physik, Universität Regensburg, \\ Federal Republic of Germany \\ Received May 22, 1987; revised version August 6, 1987
}

\begin{abstract}
The imaginary part of the optical potential for nuclear ion-ion scattering in the energy range $20 \mathrm{MeV} \lesssim E / A \lesssim 200 \mathrm{MeV}$ is derived using Feshbach's projection formalism. It is defined as the effective absorptive potential in the projected one-body Schroedinger equation for the relative motion of the colliding nuclei. Calculations are done in the ThomasFermi approximation, which accounts in a simple way for all phase-space effects as well as for the finite size of the ions. Intrinsic excitations are considered to be of one particle - one hole type in either of the ions, the other remaining in its ground state. The effective two-body interaction is taken to be of finite range. Further simplifications of the model consist in neglecting antisymmetrization between the mutual wave functions of the two ions and in the omission of the Coulomb energy.
\end{abstract}

PACS: $24.10 . \mathrm{Ht} ; 25.70 . \mathrm{Cd}$

\section{Introduction}

This article deals with the calculation of the imaginary part of the optical potential for nuclear heavy ion scattering.

The relevant collective degree of freedom is chosen to be the relative distance between the centers of mass of the two ions. This is the appropriate coordinate in a description of peripheral collisions at incident energies well above the Coulomb barrier as well as above the excitation energy of giant resonance modes. In the framework of Feshbach's projection formalism [1] one expects then that the $Q$-space (the space of all intrinsic excitations) can be considerably simpliefied. We consider here excitations of the lowest order, namely only those channels which involve at most the excitation of one hole and one particle in either nucleus, leaving the other in its ground state. Reactions involving simultaneous excitations of the two nuclei are much less important than those in which only one of the nuclei is excited. This has been shown in a recent theoretical work [2].

Furthermore we study only incoherent processes. We find that these account already for about a half of the measured reaction cross section, leaving the rest for other processes such as coherent effects at low energies and higher many particle - many hole $(m p-m h)$ excitations at higher energies, and break-up reactions. A similar result has been obtained in the case of the nucleon-nucleus potential [3], where the same effective force and the same methods were used as in our case. Furthermore there is experimental indication that coherent processes contribute in a minor way to the reaction cross section [4].

We use semiclassical methods to evaluate the imaginary part of the optical potential. They prove to be very successful in the description of $m p-m h$ level densities [5], as well as in the formulation of the real and imaginary parts of the nucleon-nucleus optical potential $[3,6]$ and of the temperature dependence of the nucleon mean free path [7].

The semiclassical approach used here is based upon the Wigner-Kirkwood expansion of the onebody density matrixy (see $[8,9]$ for an introduction to the formalism). It has been extensively studied in the evaluation of static properties of nuclei, and precise statements can be made about its validity in the Hartree-Fock framework [9]: one obtains with the semicalssical approximation exactly the average part of the microscopical Hartree-Fock results, leaving out 
the shell effects. Whereas the selfconsistent semiclassical evaluation of average nuclear energies and densities necessitates the inclusion of second and fourth order corrections in $\hbar$, these can be ignored to a very good degree of approximation when studying small deviations from the Hartree-Fock ground state due to particle-hole excitations. Even in the lowest semiclassical order, i.e. at the Thomas-Fermi level, the Pauli exclusion principle can be treated correctly (which becomes particularly transparent in phase space); finite size (i.e. nuclear surface) effects and finite-range residual forces can be incorporated in a simple and natural way. This has been demonstrated in the successful calculation of $m p-n h$ level densities [5] and their above-mentioned applications $[3,6,7]$. Preliminary results for the ion-ion potential obtained in this framework have been presented in [10].

This decade has given rise to a series of interesting studies about the imaginary part of the ion-ion potential. A formulation quite close to ours is the recent work of [2]. There, too, the optical potential is defined in a projected Schroedinger equation for the wave function of relative motion, and the total many-body wave function of the system is expanded into a product of eigenstates of the intrinsic unperturbed Hamiltonians of the two ions. At this general stage, the optical potential is still too difficult to calculate. The type of approximations and methods we then use are quite different from the ones in [2]. While we work in 2nd quantization and truncate the excitations at the $1 p-1 h$ level and then use semiclassical methods, whose merits we have outlined above, in [2] the excitations are not truncated. Instead, the propagator is handled in eikonal approximation and the excitation energies of the nuclei set constant and closure performed.

Other authors [11-13] have derived the imaginary part of the optical potential due to inelastic and transfer processes in a different context. The absorptive potential is introduced there by solving the equations of motion for the amplitudes associated with different reaction channels in a limited channel subspace. The imaginary part of the potential "mocks up" the missing channels. The ions move on classical trajectories.

Finally we quote the work inspired by the Brueckner theory of nuclear matter. An extension of the nucleon-nucleus potential [14] to the ion-ion potential has been formulated in $[15,16]$. It yields to a too weak absorptive potential. A substantial enhancement was achieved by including collective excitations in the nuclear matter potentials. This was done by modifying in a somewhat ad-hoc manner the $G$-matrix [17].

Our paper is structured as follows. In the next section we derive the optical model for relative motion, define the model space, the effective two-body interaction, and write in semiclassical approximation the expressions for the real and imaginary parts of the optical potential. In Sect. 3 we derive the expressions for the optical potential using specific density distributions of the ions. We show quantitatively that the imaginary part of the optical potential is inversely proportional to $E$ for incident energy per particle $E / A$ larger than the Fermi energy. In that section we also discuss the numerical results, specifically the spatial and energy dependence of the optical potential. In Sect. 4 we evaluate in a straight-line approximation the absorption cross section and compare it to experiment. The paper contains three appendices which fill in details of the derivations and of the momentum integrations. We conclude with a summary and outlook in Sect. 5.

\section{The Optical Model for Relative Motion}

The many-body Hamiltonian $H$ for two interacting heavy ions $A$ and $B$ with centers a distance $\mathbf{R}$ apart is

$$
\begin{aligned}
& H\left(\mathbf{r}_{1} \ldots \mathbf{r}_{A} ; \mathbf{s}_{1} \ldots \mathbf{s}_{B} ; \mathbf{R}\right) \\
& =H_{A}\left(\mathbf{r}_{1} \ldots \mathbf{r}_{A}\right)+H_{B}\left(\mathbf{s}_{1} \ldots \mathbf{s}_{B}\right)+T(\mathbf{R}) \\
& +\sum_{j=1}^{A} \sum_{i=1}^{B} v\left(\mathbf{R}, \mathbf{s}_{i}, \mathbf{r}_{j}\right)
\end{aligned}
$$

where $H_{A}$ and $H_{B}$ are the intrinsic Hamiltonians of ions $A$ and $B$ respectively, depending on the nucleon intrinsic coordinates $\mathbf{r}_{j}(j=1 \ldots A)$ and $\mathbf{s}_{i}(i=1 \ldots B)$. The interaction between a nucleon of $B$ at position $\mathbf{s}_{i}$ and one of $A$ at $\mathbf{r}_{j}$ is described by the two body operator $v\left(\mathbf{R}, \mathbf{s}_{i}, \mathbf{r}_{j}\right)$. The operator $T$ stands for the kinetic energy of relative motion.

For the many-body wave function $\Psi$, which satisfies the full Schroedinger equation $H \Psi=E \Psi$, we make the separation ansatz

$$
\begin{aligned}
& \Psi\left(\mathbf{r}_{1} \ldots \mathbf{r}_{A} ; \mathbf{s}_{1} \ldots \mathbf{s}_{B} ; \mathbf{R}\right) \\
& =\sum_{i, j} G_{i j}(\mathbf{R}) \phi_{A j}\left(\mathbf{r}_{1} \ldots \mathbf{r}_{A}\right) \phi_{B i}\left(\mathbf{s}_{1} \ldots \mathbf{s}_{B}\right)
\end{aligned}
$$

which is of very general form except for the neglect of antisymmetrization between the partial intrinsic wave functions $\phi_{A j}$ and $\phi_{B i}$, which are the eigenfunctions of $H_{A}$ and $H_{B}$, respectively. Since our interest lies mainly in higher energy reactions, where the phase spaces of the nucleons in $A$ and $B$ are quite different, this approximation is quite reasonable.

Furthermore we rewrite the two-body interaction 
$V=\sum_{j=1}^{A} \sum_{i=1}^{B} v\left(\mathbf{R}, \mathbf{s}_{i}, \mathbf{r}_{j}\right)$ in (2.1) as a sum of a mean field contribution $V_{M F}(\mathbf{R})$ between the two ions and a residual two-body interaction $V_{R}\left(\mathbf{R}, \mathbf{s}_{i}, \mathbf{r}_{j}\right)$

$V=V_{M F}(\mathbf{R})+V_{R}\left(\mathbf{R}, \mathbf{s}_{i}, \mathbf{r}_{j}\right)$

$V_{R}\left(\mathbf{R}, \mathbf{s}_{i}, \mathbf{r}_{j}\right)=V-V_{M F}(\mathbf{R})$.

It is well known that using projection techniques the many-body problem can be reduced to an effective one-body Schroedinger equation for a chosen degree of freedom [1]. Then the information about all the other degrees of freedom is accounted for formally in an effective potential, the optical potential. We choose the relevant degree of freedom to be the relative distance between ions $A$ and $B$. To obtain the optical potential for the relative motion we proceed as follows. In second quantization the expansion of the full many-body wave function (2.2) in terms of projectile and target eigenstates is expressed as

$|\Psi\rangle=\sum_{i} c_{i}\left|g_{i}\right\rangle|0\rangle+\sum_{i, j, k} c_{i j K}\left|g_{k}\right\rangle a_{j}^{+} a_{i\langle}|0\rangle+\ldots$

where $a_{j\rangle}^{+}$creates a particle above the Fermi level and $a_{i<}$ destroys a particle below the Fermi level of either ion $A$ or $B$. The ket $|0\rangle$ denotes the ground states of nuclei $A$ and $B$. The ket $\left|g_{K}\right\rangle$ is the component of the wave function which describes the relative motion and is eigenstate of $T_{\text {eff }}=T+V_{M F}$,

$T_{\text {eff }}\left|g_{k}\right\rangle=\mathrm{e}_{k}\left|g_{k}\right\rangle$.

We define projection operators $P$ and $Q$ acting on the intrinsic subspace of $A \otimes B$ as

$P=|0\rangle\langle 0|$

and

$Q=\sum_{i, j} a_{j\rangle}^{+} a_{i<}|0\rangle\langle 0| a_{i<}^{+} a_{j\rangle}+\ldots$

where $P+Q=1 ;\left[H_{A, B} ; P\right]=\left[H_{A, B}, Q\right]=0$ and also $[T, P]=[T, Q]=0$ since antisymmetrization between the wave functions of $A$ and $B$ is neglected.

We obtain the effective Schrödinger equation for the relative wave function $\left|g_{k}\right\rangle$ by operating alternatively with $P$ and $Q$ on the full Hamiltonian $H$ and eliminating $Q|\Psi\rangle$ from the two equations. Taking then the expectation value of the resulting expression in the ground state wave functions $|0\rangle$ one obtains

$$
\left\{\left(\varepsilon_{0}+T-E\right)+\left\langle 0\left|V_{\mathrm{eff}}\right| 0\right\rangle\right\} \sum_{i} c_{i}\left|g_{i}\right\rangle=0
$$

where

$V_{\mathrm{eff}}=V+V Q \frac{1}{E-H_{A}-H_{B}-T-V_{M F}-Q \widetilde{V_{R} Q+i \varepsilon}} Q V$

and $\varepsilon_{0}$ is the ground state energy of $A$ and $B$, which we set equal to zero. The residual interaction $V_{R}$ is defined in (2.3).

\subsection{The Model Space}

In practice it is of course not possible to treat the effective potential exactly. We recur to the following approximations:

(a) we neglect residual interactions $Q V_{R} Q$.

(b) we truncate the $Q$-space at the one particle - one hole $(1 p-1 h)$ level.

The neglect of residual interactions looks formally like the effective potential to 2 nd order in the twobody interaction, with an effective kinetic energy $T_{\text {eff }}$. However, by keeping $V_{M F}$ in the propagator, approximation (a) is much more powerful than the usual 2nd order expansion, which sets $Q V Q=0$, i.e. also $V_{M F}=0$.

Approximation (b) is less obvious and done mainly for calculational purposes. We expect however that in peripheral collisions most of the excitations will be of the $1 p-1 h$ type. We recall also that in the case of the nucleon-nucleus optical potential the truncation of $Q$ at the $1 p-1 h$ excitations is a very good approximation, since it leads to very reasonable values of the nucleon mean free path [7].

\subsection{The Optical Potential}

With the simplifications ( $a$ ) and ( $b$ ) we calculate matrix elements $\widetilde{V}_{\text {eff }}=\left\langle 0\left|V_{\text {eff }}\right| 0\right\rangle$ which describe the transition of the relative wave function from state $\left|g_{k^{\prime}}\right\rangle$ to state $\left|g_{k^{\prime \prime}}\right\rangle$. By inserting complete sets (in the truncated $Q$-space) of eigenfunctions of $H_{A}+H_{B}+T_{\text {eff }}$ to the left and right of the propagator in (2.7), one obtains the optical potential

$$
\begin{aligned}
V_{k^{\prime}, k^{\prime}}^{\mathrm{opt}}= & \left\langle g_{k^{\prime \prime}}\left|\widetilde{V}_{\mathrm{eff}}\right| g_{k^{\prime}}\right\rangle \\
= & \sum_{i\langle, j\rangle}\left\langle g_{k^{\prime \prime}}\left|v_{j i i j}\right| g_{k^{\prime}}\right\rangle \\
& +\sum_{v=A, B} \sum_{i\langle, j\rangle, k}\left\langle g_{k^{\prime \prime}}\left|\sum_{k} v_{i l l j}\right| g_{k}\right\rangle \\
& \cdot \frac{1}{E-\mathrm{e}_{j}^{v}+\mathrm{e}_{j}^{v}-\mathrm{e}_{k}+i \varepsilon}\left\langle g_{k}\left|\sum_{r\left\langle v_{j r r}\right|}\right| g_{k^{\prime}}\right\rangle .
\end{aligned}
$$


Here $e_{j}^{y}$ and $e_{i}^{y}$ are the particle and hole energies in either ion $\nu=A$ or $B, \mathrm{e}_{k}$ is eigenvalue to $T_{\text {eff }}$. The quantities $\vartheta_{m n m^{\prime} n^{\prime}}$ are the antisymmetrized matrix elements of the two body interaction

$V=\sum_{m, n, m^{\prime}, n^{\prime}} a_{m}^{+} a_{n}^{+} v_{m n m^{\prime} n^{\prime}} a_{n^{\prime}} a_{m^{\prime}}$,

where

$v_{m n m^{\prime} n^{\prime}}=\left\langle m n|v| m^{\prime} n^{\prime}\right\rangle=\left\langle m n|v| n^{\prime} m^{\prime}\right\rangle$

and

$\left\langle m n|v| m^{\prime} n^{\prime}\right\rangle=$

$\int \mathrm{d} 1 \mathrm{~d} 2 \mathrm{~d} 3 \mathrm{~d} 4 \varphi_{m}^{*}(1) \varphi_{n}^{*}(2) v(1,2,3,4) \varphi_{m^{\prime}}(3) \varphi_{n^{\prime}}(4)$.

Here the $\varphi_{i}$ are single particle wave functions.

The first summation in (2.8) describes the change in the relative wave function caused by the mean field $v_{j i i j}$. The second term describes the change in relative motion when $1 p-1 h$ excitations occur in one of the ions (indices $i, j$ of $v$ ), leaving the other ion inert (repeated indices of $v$, which also have to be summed over) followed by propagation and deexcitation.

\subsection{The Effective Interaction}

We neglect exchange effects in $v_{m n m^{\prime} n^{\prime}}$ and take an effective local Wigner force instead $[3,6]$. In coordinate space it reads

$$
\begin{aligned}
& v\left(\mathbf{r}_{1}+\mathbf{R}_{A}, \mathbf{r}_{2}+\mathbf{R}_{A} ; \mathbf{s}_{1}+\mathbf{R}_{B}, \mathbf{s}_{2}+\mathbf{R}_{B}\right) \\
& \quad=\delta\left(\mathbf{r}_{1}-\mathbf{r}_{2}\right) \delta\left(\mathbf{s}_{1}-\mathbf{s}_{2}\right) u\left(\mathbf{R}+\mathbf{r}_{1}-\mathbf{s}_{1}\right)
\end{aligned}
$$

where $\mathbf{R}_{A}$ and $\mathbf{R}_{B}$ are distances from the common center of mass of ions $A$ and $B$ to their respective centers, i.e. $\mathbf{R}=\mathbf{R}_{A}-\mathbf{R}_{B}$ and $\mathbf{r}_{j}, \mathbf{s}_{i}$ are intrinsic coordinates as before. The finite range two-body force $u$ has been fitted to the s-wave part of the Gogny force [18]

$u(\mathbf{r})=-u_{0} \exp \left(-r^{2} / r_{0}^{2}\right)$

of strength $u_{0}=26.5 \mathrm{MeV}$ and range $r_{0}=2.25 \mathrm{fm} \mathrm{[3,}$ 6]. It is especially designed to describe pairing properties [19] which include the same matrix elements as the ones considered in the $Q$-space of our model. The mean field part of $V^{\text {opt }}$ has a quite different type of effective vertex, which must be treated accordingly. For the mean-field vertex we use the same form for the effective interaction as given by (2.12) but the parameters are chosen such that a binding energy per particle $\varepsilon=-8 \mathrm{MeV}$ is reproduced for nucleons of one nucleus in the field of the other. We take the depth $u_{0}^{\prime}=42.7 \mathrm{MeV}$ and the range $r_{0}^{\prime}=1.4 \mathrm{fm}$. The value for the range comes again from a fit using the Gogny force [3]. Although this is a very simple parametrization of the mean-field potential, we think it is enough to describe properly the imaginary part of the optical potential, where it enters only in the propagator.

With the parameters $u_{0}$ and $r_{0}$ chosen above, good agreement with phenomenological fitted optical potential depths in nucleon-nucleus interactions has been obtained for incident energies $15 \mathrm{MeV}$ $<E<60 \mathrm{MeV}$.

The parametrization (2.12) means the following. The excitation of the particle in the excited nucleus occurs at the same place as the excitation of the hole, given by one of the Dirac delta functions. The other delta function puts the virtual excitations in the "inert nucleus" (repeated indices in the matrix element of $v$, in (2.8)) at a single point. The finite range force $u(2.13)$ is a measure for the nonlocality between these two types of excitations. In a similar way (2.12) describes the nonlocality between virtual excitations in each of the ions, for reactions in which both ions remain in the ground state.

\subsection{Semiclassical Description of the Optical Potential}

The space representation of the mean field contribution to the optical potential, the first term in (2.8), is (using (2.12))

$V_{M F}(\mathbf{R})=\int \mathrm{d} \mathbf{r}_{1} \mathrm{~d} \mathbf{s}_{2} \rho\left(\mathbf{r}_{1}\right) u\left(\mathbf{R}+\mathbf{r}_{1}-\mathbf{s}_{2}\right) \rho\left(\mathbf{s}_{2}\right)$,

where

$\rho(\mathbf{r})=\sum_{i<} \varphi_{i}^{*}(\mathbf{r}) \varphi_{i}(\mathbf{r})=\langle\mathbf{r}|\hat{\rho}| \mathbf{r}\rangle$

is the single-particle density for one ion, and $\hat{\rho}$ is the density operator. Equation (2.14) is the well known folding potential $[20]$.

Since the effective force $u$ is real, the first order contribution to $V^{\mathrm{opt}}$ is also real. This is in contrast to the effective interaction at high energies, $E / A \gtrsim$ $500 \mathrm{MeV}$, which is complex [21].

Therefore we calculate only the imaginary part of the second contribution to $V^{\text {opt }}$, which we call $V_{2}$ assuming that its real part will be a small correction to $V_{M F}$.

The space representation of the imaginary part of $V^{\text {opt }}$ is then 
$\operatorname{Im} V_{2}^{\mathbf{R}, \mathbf{R}^{\prime}}=\sum_{\nu=A, B} \int \mathrm{d} t \mathrm{e}^{\mathrm{i} E t}\left\langle\mathbf{R}\left|\hat{\rho}_{M} \mathrm{e}^{-\mathrm{i}\left(T+\boldsymbol{V}_{M \boldsymbol{F}}\right) t}\right| \mathbf{R}^{\prime}\right\rangle$

$\cdot \int \mathrm{d} \mathbf{r}_{1} \int \mathrm{d} \mathbf{r}_{1}^{\prime}\left\langle\mathbf{r}_{1}\left|\hat{\rho} \mathrm{e}^{\mathrm{i} H_{v} t}\right| \mathbf{r}_{1}^{\prime}\right\rangle\left\langle\mathbf{r}_{1}\left|\hat{\bar{\rho}} \mathrm{e}^{-\mathrm{i} H_{v} t}\right| \mathbf{r}_{1}^{\prime}\right\rangle$

$\iint \mathrm{d} \mathbf{s}_{2} \rho\left(\mathbf{s}_{2}\right) u\left(\mathbf{R}+\mathbf{r}_{1}-\mathbf{s}_{2}\right) \int \mathrm{d} \mathbf{s}_{2}^{\prime} \rho\left(\mathbf{s}_{2}^{\prime}\right) u\left(\mathbf{R}^{\prime}+\mathbf{r}_{1}^{\prime}-\mathbf{s}_{2}^{\prime}\right)$

where we have Fourier transformed the propagator in (2.8). The particle density operator is represented by $\hat{\bar{\rho}}$ and the hole density operator by $\hat{\rho}$. The density associated with relative motion is denoted by $\hat{\rho}_{M}$. The unprimed and primed coordinates denote the interaction points before and after propagation, respectively. Before discussing the semiclassical approximation, it is instructive to compare (2.16) to the imaginary part of the nucleon-nucleus optical potential, derived in [6] by use of similar assumptions. Equation (2.16) reduces to the nucleon-nucleus optical potential by replacing the density of the inert ion with $\rho(\mathbf{s}) \propto \delta(\mathbf{s})$, i.e. making it a point particle, and by interpreting the coordinates $\mathbf{R}, \mathbf{R}^{\prime}$ as the coordinates of the extra (incident) nucleon. Then $T+V_{M F}$ is just the Hamiltonian of the extra particle, which can be above the Fermi level $\left(\hat{\rho}_{M} \rightarrow \hat{\rho}\right)$ or below $\left(\hat{\rho}_{M} \rightarrow \hat{\rho}\right)$. In the first case one obtains the polarization graph $(2 p-1 h)$, in the second the correlation graph $(1 p-2 h)$.

We now proceed to write the imaginary part of the optical potential in semiclassical approximation. In this approximation the brackets in (2.16) become

$$
\begin{aligned}
& \left\langle\mathbf{r}_{1}\left|\hat{\rho} \mathrm{e}^{\mathrm{i} H t}\right| \mathbf{r}_{1}^{\prime}\right\rangle \\
& =\int \frac{d \mathbf{p}}{(2 \pi)^{3}} \mathrm{e}^{\mathrm{i} \mathbf{p} \cdot \mathbf{x}} \mathrm{e}^{\mathrm{it}\left(\frac{\mathbf{p}^{2}}{2 m}+V_{0}(\mathbf{X})\right)} \Theta\left(\lambda-\frac{\mathbf{p}^{2}}{2 m}-V_{0}(\mathbf{X})\right)
\end{aligned}
$$

and similarly for the bracket containing the particle density $\hat{\bar{\rho}}$, and

$$
\begin{aligned}
& \left\langle\mathbf{Y}+\frac{\mathbf{y}}{2}\left|\hat{\rho}_{M} \mathrm{e}^{-\mathrm{i}\left(T+V_{M F}\right) t}\right| \mathbf{Y}-\frac{\mathbf{y}}{2}\right\rangle \\
& =\int \frac{\mathrm{d} \mathbf{p}}{(2 \pi)^{3}} \mathrm{e}^{-\mathrm{i} \mathbf{p} \cdot \mathbf{y}} \mathrm{e}^{-\mathrm{i} t}\left(\frac{\mathbf{p}^{2}}{2 \mu}+V_{M F}(\mathbf{Y})\right)
\end{aligned}
$$

\section{for the relative motion}

In (2.17) the step function $\Theta$ restricts the holes to be below the Fermi energy and $V_{0}(\mathbf{X})$ is the potential of one of the ions in the intrinsic system (e.g. a Harmonic Oscillator or Woods-Saxon potential).

The coordinates $\mathbf{X}$ and $\mathbf{x}$ are the mean and relative distance, respectively, of the coordinates $\mathbf{r}_{1}$ and $\mathbf{r}_{1}^{\prime}$, $\mathbf{X}=\left(\mathbf{r}_{1}+\mathbf{r}_{1}^{\prime}\right) / 2, \quad \mathbf{X}=\mathbf{r}_{1}-\mathbf{r}_{1}^{\prime}, \mu$ is the reduced mass of ions $A$ and $B$ and $\mathbf{Y}=\frac{\mathbf{R}+\mathbf{R}^{\prime}}{2}, \mathbf{y}=\mathbf{R}-\mathbf{R}^{\prime}$.

With definitions (2.17) and (2.18) and the Gaussian interaction (2.13) we write the Wigner transform of the imaginary part of the optical potential, $W$, with
$Q$ the conjugate momentum to $Y$, as

$$
\begin{aligned}
& W(E, \mathbf{Y}, \mathbf{Q})=\frac{u_{0}^{2}}{(2 \pi)^{5}}\left(\frac{\pi r_{0}^{2}}{\sqrt{2}}\right)^{3 / 2} \int \mathrm{d} \mathbf{X} \int \mathrm{d} \mathbf{p}_{0} \int \mathrm{d} \mathbf{p} \int \mathrm{d} \mathbf{p}^{\prime} \\
& \delta\left(\mathbf{Q}-\mathbf{p}_{0}+\mathbf{p}-\mathbf{p}^{\prime}\right) \delta\left(E-\frac{p_{0}^{2}}{2 \mu}-\frac{p^{\prime 2}}{2 m}+\frac{\mathbf{p}^{2}}{2 m}-V_{M F}(\mathbf{Y})\right) \\
& \Theta\left(\lambda-\frac{\mathbf{p}^{2}}{2 m}-V_{0}(\mathbf{X})\right) \Theta\left(\frac{\mathbf{p}^{\prime 2}}{2 m}+V_{0}(\mathbf{X})-\lambda\right) \\
& \exp \left(-\frac{r_{0}^{2}}{2}\left(\mathbf{p}-\mathbf{p}^{\prime}\right)^{2}\right) \mathscr{F}\left[\mathbf{Y}+\mathbf{X}, \mathbf{Q}-\mathbf{p}_{0}\right]
\end{aligned}
$$

where

$$
\begin{aligned}
& \mathscr{F}\left[\mathbf{Y}+\mathbf{X}, \mathbf{Q}-\mathbf{p}_{0}\right]=\int \mathrm{d} \xi \mathrm{e}^{-\frac{2}{r_{0}^{2}}\left(\mathbf{Y}+\mathbf{X}-\xi^{\prime}\right)^{2}} \\
& \cdot \int \mathrm{d} \xi \mathrm{e}^{-\mathrm{i} \xi \cdot\left(\mathbf{Q}-\mathbf{p}_{0}\right)} \rho\left(\xi^{\prime}-\boldsymbol{\xi} / 2\right) \rho\left(\xi^{\prime}+\xi / 2\right)
\end{aligned}
$$

contains the nuclear structure information in the Wigner transform of the product of the nuclear densities of the inert ion at different spatial points. The two delta functions in (2.19) describe momentum and energy conservation, the $\Theta$ functions take the phase space for particles and holes into account. In (2.19) the momenta are as follows. The particle momentum is denoted by $\mathbf{p}$, the hole momentum by $\mathbf{p}^{\prime}$ and $\mathbf{p}_{0}$ is the momentum of relative motion, conjugate to the distance $\mathbf{y}=\mathbf{R}-\mathbf{R}^{\prime}$.

Comparing (2.19) to the corresponding formula for the polarization graph of the nucleon-nucleus optical potential [6] we note the following. In that case an additional step function $\Theta\left(\frac{\mathbf{p}_{0}^{2}}{2 m}+V_{M F}(\mathbf{Y})-\lambda\right)$ requires the impinging particle to be above the Fermi level, due to the Pauli principle. However, at incident momentum $\mathbf{Q}$ well above the Fermi momentum, this step function becomes less important. The reason is that contributions to the $\mathbf{p}_{0}$-integration coming from small $\mathbf{p}_{0}, \frac{\mathbf{p}_{0}^{2}}{2 m}<\lambda-V_{M F}(\mathbf{y})$, are suppressed by the finite range force $\exp \left(-\frac{r_{0}^{2}}{2}\left(\mathbf{p}-\mathbf{p}^{\prime}\right)^{2}\right)$. Qualitatively this can be seen from the following consideration. The momentum transfer to the particle-hole excitation is $\mathbf{p}^{\prime}$ $-\mathbf{p}=\mathbf{Q}-\mathbf{p}_{0}$, by virtue of the momentum delta function. This means that for small $\mathbf{p}_{0}$ the momentum transfer is large and is suppressed by the gaussian form of the finite range force. In other words: a large slow-down of the relative motion is relatively improbable. The suppression increases with the mass of the projectile, since $\mathbf{Q}$ increases with the effective mass of the system. The neglect of the relative antisymmetrization between the two ions is thus justified for not too light projectiles. The Fermi spheres of the 
two nuclei are not likely to overlap during the reaction.

To make the argument more quantitative we calculate the suppression factor $\exp \left(-\frac{r_{0}^{2}}{2}\left(\mathbf{p}-\mathbf{p}^{\prime}\right)^{2}\right)$ for the overlap of the Fermi sphere(s) at an incident momentum corresponding to a $20 \mathrm{MeV}$ nucleon. Whereas in the reaction $n+{ }^{12} \mathrm{C}$ this factor can be as big as 0.7 , it is always smaller than $2 \times 10^{-2}$ or $2 \times 10^{-7}$ in the ion-ion reactions $\alpha+{ }^{12} C$ and ${ }^{12} C+{ }^{12} C$, respectively.

\subsection{Density Distributions}

For the explicit evaluation of the optical potential we need to know the density distributions of the two interacting ions. We parametrize the ion densities to be either of Gaussian shape (for light ions) or WoodsSaxon distributions (for heavier ions). We have to resort to further approximations in the case of the imaginary part of the optical potential for WoodsSaxon distributions, since the number of integrations, which remain after integrating the delta functions in (2.18), is too large to be solved numerically. The approximation consists in substituting the $\mathbf{X}$-dependence in $V_{0}(\mathbf{X})$ by the fixed value $\mathbf{Y}$, which is the relative distance between the two ions. This approximation was introduced first by Hasse and Schuck [6] in the evaluation of the nucleon-nucleus optical potential, and designated as "local momentum approximation" (LMA). In that case the approximation was reasonable in the interior of the nucleus, but the resulting imaginary part of the optical potential dropped too fast at the surface.

Using Woods-Saxon profiles together with LMA, the evaluation of the imaginary part $W(E, \mathbf{Y}, \mathbf{Q})$ of the optical potential is reduced to a 1-dimensional integral; in the case of Gaussian distributions, $W$ is expressed by a two-fold integral.

As we show later in subsection 3.4, the LMA is quite unsatisfactory in the case of ion-ion potentials. We therefore will perform most of the calculations using Gaussian density profiles. We choose the reaction ${ }^{12} C+{ }^{12} C$ for a comparison with experiment, because for ${ }^{12} \mathrm{C}$ a Gaussian density is presumably reasonable.

Since a Woods-Saxon type of expression can be well parametrized as a superposition of Gaussians [22], the imaginary part of the optical potential can be readily obtained also for reactions involving heavier nuclei, without the need of LMA. We plan to make a systematic analysis of reactions involving nuclei of light and heavy masses in a later work.

\section{Calculations}

In the following subsections we discuss the results for the mean field and imaginary part of the optical potential. All calculations are performed for a symmetric system $A=B$, i.e. for collisions involving nuclei of the same mass.

\subsection{The Ion-Ion Mean Field}

a) Gaussian Densities. The mean field can be readily obtained for Gaussian density distributions for both ions

$\rho=\rho_{0} \mathrm{e}^{-r^{2} / a^{2}}$

using (2.14). The result is in Hartree approximation

$V_{M F}(Y)=A^{2} u_{0}^{\prime}\left(\frac{r_{0}^{\prime 2}}{2 a^{2}+r_{0}^{\prime 2}}\right)^{3 / 2} \exp \left(-Y^{2} /\left(2 a^{2}+r_{0}^{2 \prime}\right)\right)$,

where $A$ is the particle number of one of the ions, $\rho$ is normalized to $A$ and $a^{2}$ are chosen such that the root mean square radius is reproduced

$\left\langle r^{2}\right\rangle^{1 / 2}=1.2 A^{1 / 3}=\left(\frac{3}{2} a^{2}\right)^{\frac{1}{2}}$

b) Symmetrized Woods-Saxon Densities. A symmetrized Woods-Saxon density [23], for each ion normalized to the particle number $A$, takes the form

$$
\rho=\rho_{0} \frac{1}{\cosh \left(\frac{R}{a}\right)+\cosh \left(\frac{r}{a}\right)}
$$

with

$\rho_{0}=\frac{3 A}{3 \pi a^{3}}\left(\frac{R^{3}}{a^{3}}+\pi^{2} \frac{R}{a}\right)^{-1} \sinh \left(\frac{R}{a}\right)$,

where $a$ is the surface thickness and $R$ is a measure of the root mean square radius of the ion. In this case the integrations in (2.14) cannot be performed in closed form.

In Fig. 1 we compare $V_{M F}(Y)$ as function of the relative distance $Y$ between the two ions, for Gaussians (dashed line) and symmetrized Woods-Saxon distributions (full line) of the densities, taking $A=64$.

We observe that the central mean field energy per particle is for Gaussians about $45 \mathrm{MeV}$ and for the Woods-Saxons about $56 \mathrm{MeV}$. In the latter case it is about $10 \%$ higher than the usually accepted depth of $50 \mathrm{MeV}$. 


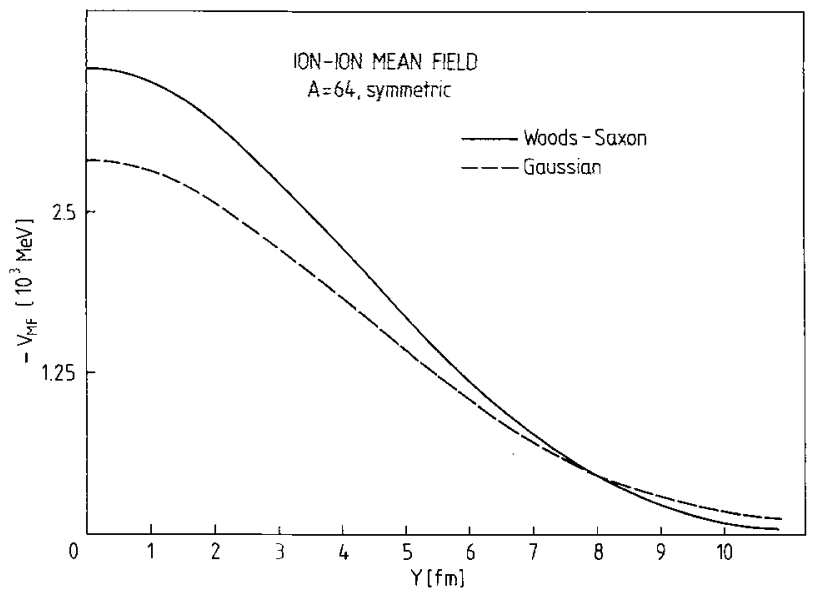

Fig. 1. Ion-ion mean field $V_{M F}(Y)$ as function of the mean distance $Y$ between the ions, calculated for Gaussian density profiles (dashed line) and symmetric Woods-Saxon densities (solid line)

\subsection{The Imaginary Part of the Optical Potential}

The momentum integrations in (2.19) can most conveniently be performed by defining new coordinates $\mathbf{q}=\mathbf{p}-\mathbf{p}^{\prime}$ and $\mathbf{P}=\frac{\mathbf{p}+\mathbf{p}^{\prime}}{2}$. The nine-fold momentum integrations can be reduced to a one-dimensional one for both density profiles, Woods-Saxon together with LMA, or Gaussians. This is done in Appendix B. All results are for the on shell condition $E=\frac{Q^{2}}{2 \mu}$
$+V_{M F}(Y)$. For Gaussians we obtain

$$
\begin{aligned}
W^{G}(E(Q), Y)= & \frac{C}{Q Y} \int_{0}^{c_{t}} \mathrm{~d} X X \mathrm{e}^{-\frac{2}{a^{2}+r_{0}^{2}}\left(Y^{2}+X^{2}\right)} \\
& \cdot 2 \sinh \left(\frac{4 X Y}{a^{2}+r_{0}^{2}}\right) \int_{0}^{\infty} \mathrm{d} q q \\
& \cdot \mathrm{e}^{-\frac{q^{2}\left(r_{0}^{2}+a^{2}\right)}{2}} I(q, X),
\end{aligned}
$$

where $c_{t}$ denotes the classical turning point for $V_{0}(X)$, $I(q, X)$ is defined in the expressions in curly brackets of (B.17) in Appendix B and

$$
C=\frac{A^{2} r_{0}^{6} \mu u_{0}^{2}}{16\left(2^{3 / 2} \pi\left(a^{2}+r_{0}^{2}\right)\right)^{1 / 2}} .
$$

For the symmetrized Woods-Saxon densities we obtain in the LMA

$$
\begin{gathered}
W^{W \cdot S .}(E(Q), Y)=\frac{C_{1}}{Q} \int_{0}^{\infty} \mathrm{d} q q \mathrm{e}^{-\frac{q^{2} r_{0}^{z}}{2}} I(q, Y) \\
\cdot\left\{\frac{R \cos (q, R)-\frac{a \pi \sin (q R) \cosh (a \pi q)}{\sinh (a \pi q)}}{q^{2} \sinh ^{2}(a \pi q)}\right\},
\end{gathered}
$$

where

$C_{1}=\frac{2 \pi^{2} a^{2} r_{0}^{6} u_{0}^{2} \mu}{(2 \sqrt{2})^{3 / 2}}\left(\frac{3 A}{4 a^{3}}\left(\frac{R^{3}}{a^{3}}+\pi^{2} \frac{R}{a}\right)^{-1}\right)^{2}$.

This is a 1-fold integration, in contrast to (3.6) for Gaussians, due to the LMA.

\subsection{Asymptotic Behaviour of $W(E(Q), Y)$ for Large $E$}

At large incident energy per particle, $E / A \gtrsim \lambda$, the imaginary part $W$ is inversely proportional to the momentum $Q$. This is a useful relation, because it allows to deduce the $E$-dependence of $W$ in the energy region $E / A>\lambda$ from a single value of $W$. We derive this dependence in Appendix C.

\subsection{Numerical Results for $W(E(Q), Y)$}

For all calculations in this subsection we take a symmetric reaction with $A=64$. The particular choice of $A=64$ is arbitrary and irrelevant for the discussion which follows. The parameters of the two-body interaction are $u_{0}=26.5 \mathrm{MeV}$ and $r_{0}=2.25 \mathrm{fm}$, as mentioned in Sect. 2.3.

a) Spatial Dependence. First we discuss the results of $W$ as a function of the distance between the two ions. In Fig. 2 we compare the behaviour of $W$ at fixed energy $E / A=150 \mathrm{MeV}$ for Gaussian density distributions of the two ions. We either take the ion-ion mean-field $V_{M F}$ in the propagator of Eq. (2.7) in account, or set it to zero. We also show the results for the local momentum approximation LMA. The full calculation in presence of the mean-field yields smaller values of $W$ as compared to a calculation with $V_{M F}=0$, especially for small values of the mean distance $Y$. The LMA calculation is, at least for Gaussians, unsatisfactory. There is a sharp cutoff at the classical turning point, so that the long-tail behaviour is suppressed and because of normalization the central part is overestimated. The spatial dependence keeps its qualitative behaviour when the energy is changed, see Fig. 3 for Gaussian densities. Here, the mean-field is taken in account. In Fig. 4 we show the spatial dependence for symmetrized Woods-Saxons in LMA with $E / A=75 \mathrm{MEV}$, solid line. For comparison, we include a curve for Gaussian distributions, in LMA (dashed line) as well as the exact calculation (dash-dotted). The results in Fig. 4 are with $V_{M F}$ turned off. We observe in Figs. 2-4 that for Gaussian 


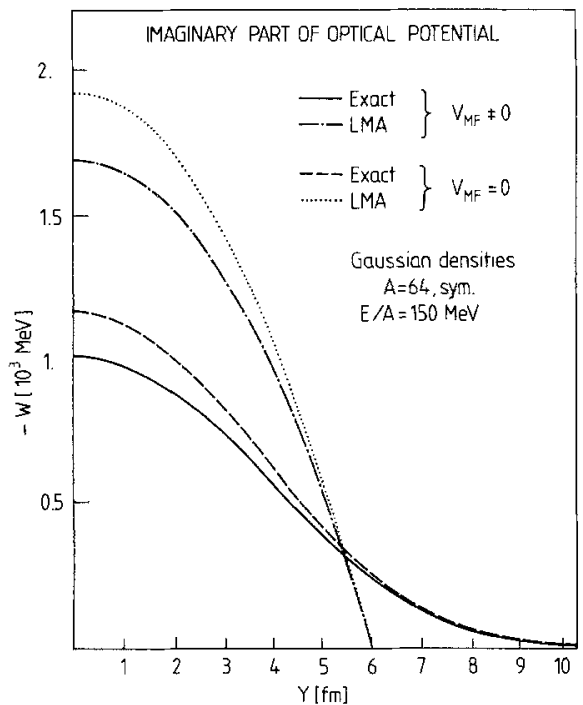

Fig. 2. Spatial dependence of the imaginary part of the optical potential $W(E, Y)$ at fixed incident energy per particle $E / A=150 \mathrm{MeV}$ for Gaussian densities of the ions. The effect of $V_{M F}$ is shown (solid line) as compared to $W$ calculated without $V_{M F}$ (dashed line). The local momentum approximation (LMA) is illustrated for the cases above, dotted line if $V_{M F}=0$, dash-dotted line if $V_{M F}$ is included

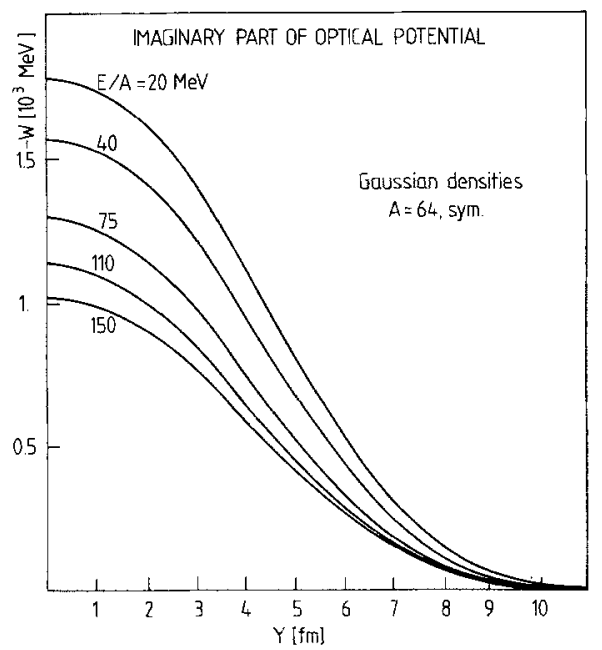

Fig. 3. $W(E, Y)$ as function of the distance $Y$ between the two ions with the incident energy $E / A$ as parameter, for Gaussian distributions and with $V_{M F}$ included

densities, $W$ has also a Gaussian shape and for Woods-Saxons, $W$ looks approximately like a Woods-Saxon too.

b) Energy Dependence. The influence of the meanfield potential on $W$ is shown in Fig. $5 \mathrm{a}$, at a fixed distance $Y=5.5 \mathrm{fm}$, using Gaussian densities. The effect of the mean-field is to shift the energy scale, since it produces an effective acceleration of the nuclei to-

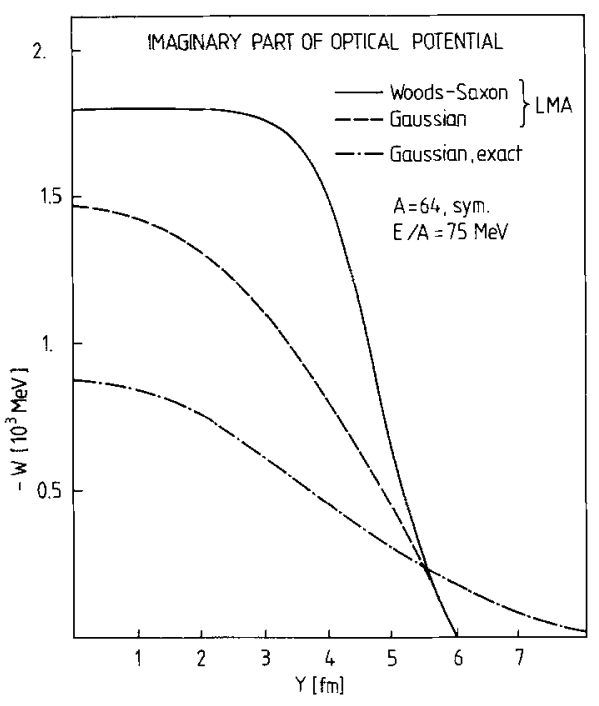

Fig. 4. Spatial dependence of $W(E, Y)$ at fixed energy $E / A=75 \mathrm{MeV}$ for different density profiles. Full line - Woods-Saxon density in LMA; dashed line - Gaussian density in LMA; dash-dotted line - Gaussian exact
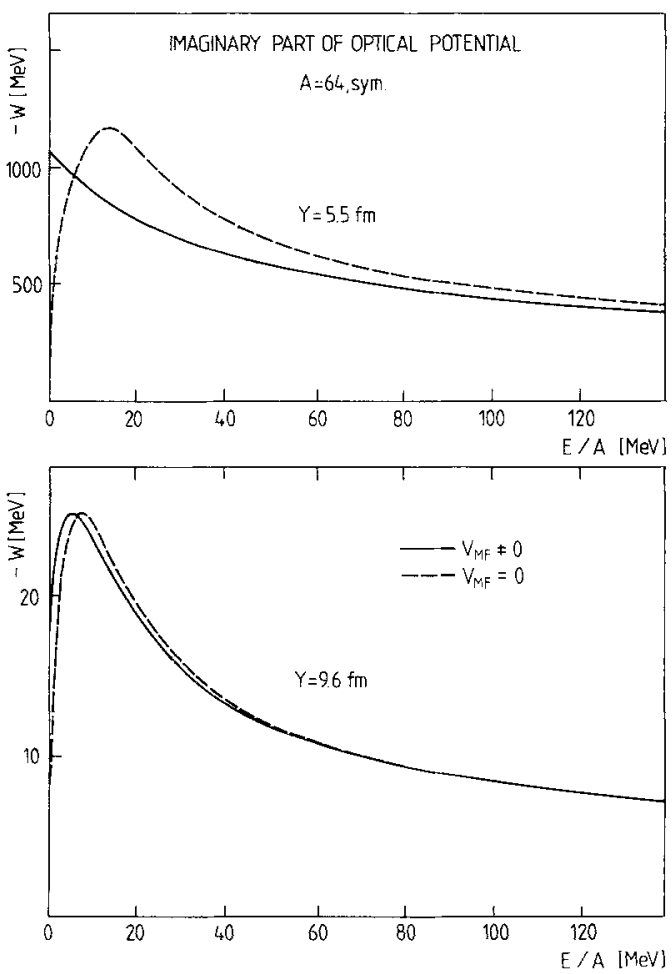

Fig. 5a. Energy dependence of $W(E, Y)$ for a fixed distance $Y=5.5 \mathrm{fm}$ between the ions, for Gaussian densities. Full line $-V_{M F}$ included; dashed line - no $V_{M F}, \mathbf{b}-$ Same as in a, but for $Y=9.6 \mathrm{fm}$

wards each other during the approach. At larger distances, the mean-field is weaker and therefore has almost no influence on $W$ (see Fig. $5 \mathbf{b}$ with $Y=9.6 \mathrm{fm}$ ).

Since the calculation of $W$ involves too many inte- 


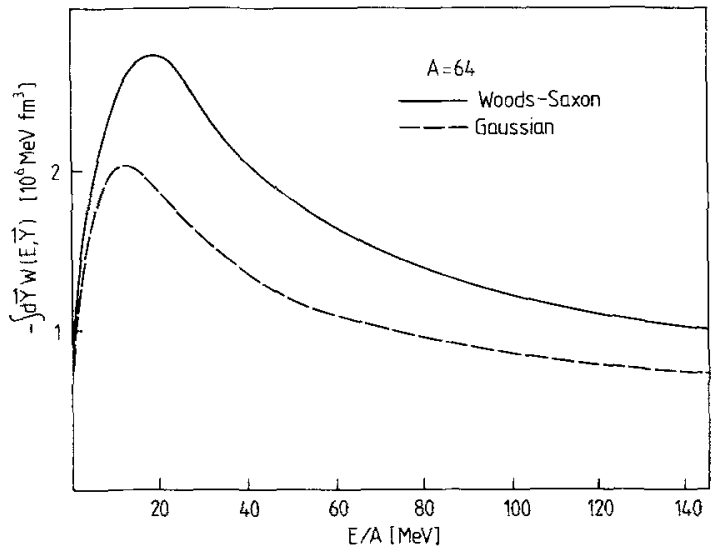

Fig. 6. The $Y$-integrated imaginary part of the optical potential, as function of $E / A$, calculated for Gaussian densities (dashed line) and sym. Woods-Saxons (solid line)

grations when we use Woods-Saxon like densities for the nuclei, it is instructive to see, how the choice of parametrization influences integrated quantities, like cross section, calculated from $W$. To get a feeling for this, we evaluate the integral $\int \mathrm{d} Y W(E(Q), Y)$ as function of the incident energy. We point out that the spatial integration of $W$ can be done exactly also in the case of Woods-Saxon densities, i.e. without recurring to the LMA. In Fig. 6 we compare the integrated absorptive potential for Gaussians and Woods-Saxons, with $V_{M F}=0$. Although the magnitude is different by a factor of about 1.3, the qualitative behaviour remains quite similar.

\section{Cross Sections}

To have a first estimate for cross sections we solve the Schroedinger equation for the ion-ion relative motion in a straight-line approximation [24], for Gaussian density distributions.

The absorption cross section $\sigma_{a b s}(E)$ is with $\mathbf{Y}$ $=(\mathbf{b}, z)$

$\sigma_{a b s}(E)=\int_{\mathbf{B}}^{\infty}\left(1-\exp \left[\frac{2}{\hbar v_{0}} \int_{-\infty}^{\infty} W(\mathbf{b}, z, E) \mathrm{d} z\right]\right) \mathrm{d}^{2} b$,

where $\mathbf{b}$ denotes the impact parameter and $v_{0}$ the incident velocity, and where $\mathbf{B}$ is a cutoff parameter, see the discussion below. As before, $W$ denotes the imaginary part of the optical potential.

The validity of the calculation is restricted at lower energies by the assumptions intrinsic to Glauber theory, and at higher energies due to the fact that relativistic effects become important. We consider the reaction ${ }^{12} \mathrm{C}+{ }^{12} \mathrm{C}$. We can state that the calculation is valid roughly in the regime $20 \mathrm{MeV} \lesssim E / A \lesssim 100 \mathrm{MeV}$.

The calculated imaginary part of the optical potential for the ${ }^{12} \mathrm{C}$ ions can be parametrized by a Gaussian with an energy dependent width. Then (4.1) is reduced to the following expression

$\sigma_{a b s}(E)=\pi \Delta^{2}(E) \int_{0}^{\gamma(E) \exp \left(-B^{2} / \Delta^{2}\right)} \frac{\mathrm{d} x}{x}\left(1-\mathrm{e}^{-x}\right)$

Here $A(E)$ is the energy ependent width of the Gaussian, the quantity $\gamma(E)$ is defined as

$\gamma(E)=2 \sqrt{\pi} \Delta H / \hbar v_{0}$

where $H$ is the absolute value of the depth of the absorptive potential. We have introduced the parameter $B=|\mathbf{B}|$ to study the contribution of different regions of impact parameters, $B$ denoting the smallest impact parameter in the integral. Certainly (4.1) is meant originally to contain $b \in[0, \infty]$. However, we include only $1 p-1 h$ excitations, which we expect to be most important for peripheral reactions. In the regions of larger overlap of the ion densities other processes, such as fragmentation, are dominant. There is of course no sharp cutoff which separates these different types of processes. In Fig. 7 we show the energy dependence of the absorptive potential for several fixed values of the mean distance $Y$ between the centers of the two ${ }^{12} \mathrm{C}$ ions. We like to point out that the results are not sensitive to how accurate the mean field $V_{M F}$ is calculated. The curves shown are almost indistinguishable from the ones calculated even with $V_{M F}=0$. In Fig. 8 we display $\sigma_{a b s}$ calculated

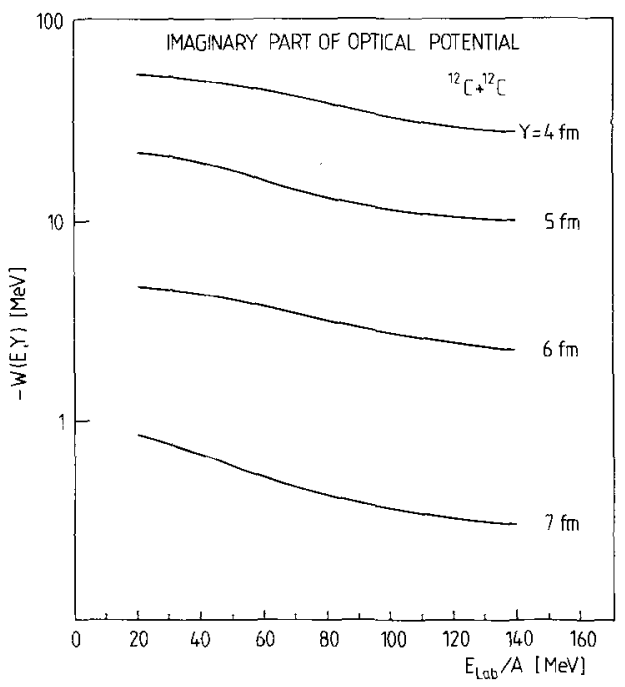

Fig. 7. The imaginary part of the optical potential for ${ }^{12} \mathrm{C}+{ }^{12} \mathrm{C}$ as function of the laboratory energy per particle $E_{\text {lab }} / A$ for different values of $Y$ 


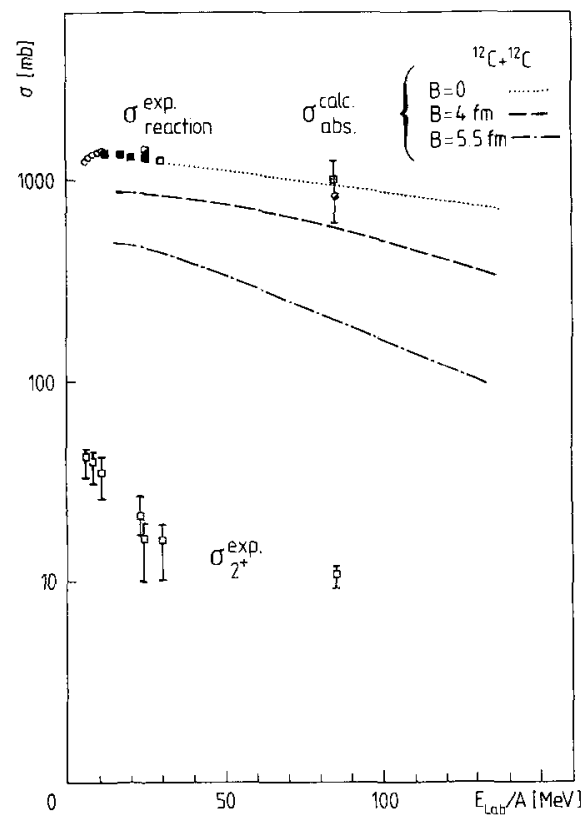

Fig. 8. Comparison of the experimental reaction cross section $\sigma_{\text {reaction }}$ with the calculated absorption cross section $\sigma_{a b s}$ (for three different impact parameter cutoffs, see Sect. 4 for the ${ }^{12} C+{ }^{12} C$ system as function of $E_{\mathrm{lab}} / A$. The experimental inelastic excitation of the $\left(J^{\Pi}, E^{*}\right)=\left(2^{+}, 4.4 \mathrm{MeV}\right)$ state of ${ }^{12} C$ is also shown, $\left.\sigma_{2^{+}}[4]\right)$

for $B=0,4$ and $5.5 \mathrm{fm}$. At $B=0$, the minimum impact parameter corresponds to two nuclei that fully overlap, at $5.5 \mathrm{fm}$ to two touching ${ }^{12} \mathrm{C}$, each with a rms radius of $2.75 \mathrm{fm}$. The calculations are compared to the experimental reaction cross section $\sigma_{\text {reaction }}$ as well as to the experimental inelastic cross section $\sigma_{2+}$ for the excitation $\left(J^{I}, E^{*}\right)=\left(2^{+}, 4.4 \mathrm{MeV}\right)$ of ${ }^{12} C$. The latter contributes only about $1 \%-2 \%$ to $\sigma_{\text {reaction }}$ [4].

We conclude that the incoherent $1 p-1 h$ excitations in our calculation exhaust to a large extent the experimental reaction cross section. For the intermediate value $B=4 \mathrm{fm}$ we obtain about $70 \%$ of the experimental reaction cross section.

\section{Summary and Conclusions}

A derivation of the imaginary part of the nucleusnucleus optical potential is presented, using a projection technique [1], where the allowed excitations are assumed to be only one particle - one hole states. We define the optical potential to be the effective potential which results when the many-body Schroedinger equation is reduced to a one-body Schroedinger equation for the relative motion of the interacting ions.

Since we employ semiclassical methods, the finite size of the ions as well as phase space factors are automatically incorporated. We use a real-valued effective two-body interaction of finite range, fitted to the Gogny force $[3,6,18]$.

The calculation of the absorptive potential is almost analytical for Gaussian density distributions. For density profiles of the Woods-Saxon type, which are more realistic than Gaussians for larger ions, the calculation of the optical potential involves too many nested integrations, which cannot be solved analytically. This is, however, not a major problem, since there exist parametrizations for Woods-Saxons as superpositions of Gaussians.

The energy domain considered is $20 \mathrm{MeV} \lesssim E / A \lesssim$ $200 \mathrm{MeV}$. Due to the neglect of the antisymmetrization between the two nuclei, our calculation becomes less reliable at lower energies $(E / A \lesssim 20 \mathrm{MeV})$ and non-peripheral collisions, since the phase space of nucleons in one and the other nucleus overlap strongly in this case. Central collisions are not well described in our model also at higher energies $(E / A \gtrsim 200 \mathrm{MeV})$, since we take only $1 p-1 h$ excitations into account and do not adjust the nuclear densities selfconsistently, but take static density profiles for each nucleus. Thus we neglect all kinds of higher-order excitation channels, deformations and break-up, of importance at small impact parameters. Therefore the best way to test our calculations is to compare to exclusive experiments, where peripheral collisions are selected. Angle-integrated cross sections, which contain integrals over all impact parameters $b$, cannot be calculated accurately. Nevertheless, the results seem to be promising because small impact parameters contribute less to the cross section due to the $\int b \mathrm{~d} b$ term in the integration. We analyze in particular the symmetric reaction ${ }^{12} C+{ }^{12} C$ at $20 \mathrm{MeV} \lesssim E / A \lesssim$ $100 \mathrm{MeV}$ and find that the incoherent superposition of $1 p-1 h$ excitations considered in our model covers already a large fraction of the measured reaction cross section. We assume hereby that the ions move on straight-line trajectories. We exclude in an approximate way central collisions by limiting the integration over impact parameters from below. If we take $4 \mathrm{fm}$ to be the minimal value for the impact parameter (the rms radius of ${ }^{12} \mathrm{C}$ is $2.75 \mathrm{fm}$ ), we obtain about $70 \%$ of the measured reaction cross section [4]. The remaining part of the reaction cross section is probably due to either coherent excitations or to incoherent multi particle - multi hole excitations - the former one being more important at lower energies and the latter at higher energies - and also to break-up reactions. The collective $2^{+}$state of ${ }^{12} \mathrm{C}$, measured in ${ }^{12} \mathrm{C}+{ }^{12} \mathrm{C}$ reactions [4], contributes only to roughly $1 \%-2 \%$ of the reaction cross section. This number suggests that the role of collective states, as compared to incoherent processes, is minor. 
We plan to improve our calculations in the following ways. (i) The trajectories of the two colliding nuclei should be corrected to include the Coulomb deflection, (ii) $1 p-1 h$ excitations in both nuclei at the same time should be included. (iii) Experimental evidence implies that elastic scattering is by no means negligeable at high energies (of the order of $1 \mathrm{GeV}$ per particle) [25]. We plan to extend our work to these energies. For that it will be necessary to correct for relativistic effects and modify the effective interaction to account for pion production, etc.

\section{Appendix A}

Here we show in detail the derivation of (2.19-20), which describe the imaginary part of the optical potential.

Using (2.16) for the space representation of the imaginary part of the optical potential and (2.17) and (2.18) for the semiclassical approximation, we derive the Wigner transform $W(E, Y, Q)$ of $(2.17)$. With the variable substitutions $\mathbf{Z}=\frac{\mathbf{y}+\mathbf{x}}{2}, \mathbf{z}=\mathbf{y}-\mathbf{x}$ we obtain

$$
\begin{aligned}
& W(E, \mathbf{Y}, \mathbf{Q})=\int \mathrm{d} \mathbf{y} \mathrm{e}^{\mathrm{i} \mathbf{y} \cdot \mathbf{Q}} \operatorname{Im} V_{2}^{\mathbf{Y}, \mathbf{y}} \\
& =\int \mathrm{d} t \mathrm{e}^{\mathrm{i} E t} \int \mathrm{d} \mathbf{z} \int \mathrm{d} \mathbf{Z} \mathrm{e}^{\mathrm{i}\left(\mathbf{Z}+\frac{\mathbf{Z}}{2}\right) \cdot \mathbf{Q}} \\
& \int \mathrm{d} \mathbf{X} \int \frac{\mathrm{d} \mathbf{p}_{0}}{(2 \pi)^{3}} \int \frac{\mathrm{d} \mathbf{p}}{(2 \pi)^{3}} \int \frac{\mathrm{d} \mathbf{p}^{\prime}}{(2 \pi)^{3}} \\
& \cdot \mathrm{e}^{-\mathrm{i} \mathbf{p}_{0}\left(\mathbf{Z}+\frac{\mathbf{z}}{2}\right)} \mathrm{e}^{\mathrm{i}\left(\mathbf{p}-\mathbf{p}^{\prime}\right) \cdot\left(\mathbf{Z}-\frac{\boldsymbol{z}}{2}\right)} \mathrm{e}^{-\mathrm{i} t\left(\frac{\mathbf{p}_{0}^{2}}{2 \mu k}+V_{M P}(\mathbf{Y})\right)} \mathrm{e}^{\mathrm{i} t\left(\frac{\mathbf{p}^{2}}{2 m}+V_{0}(\mathbf{X})\right)} \\
& \cdot e^{-i t\left(\frac{\mathbf{p}^{2 \cdot}}{2 m}+V_{0}(\mathbf{X})\right)} \\
& \cdot \Theta\left(\lambda-\frac{\mathbf{p}^{2}}{2 m}-V_{0}(\mathbf{X})\right) \Theta\left(\frac{\mathbf{p}^{\prime 2}}{2 m}+V_{0}(\mathbf{X})-\lambda\right) \\
& \cdot F(\mathbf{Y}+\mathbf{X}+\mathbf{Z}) F(\mathbf{Y}+\mathbf{X}-\mathbf{Z}),
\end{aligned}
$$

where $F$ describes an average of the two-body interaction $u$ in the one-particle density distribution $\rho(\mathbf{s})$ of the inert ion

$F(\mathbf{Y}+\mathbf{X} \pm \mathbf{Z})=\int \mathrm{d} \mathbf{s} \rho(\mathbf{s}) u(\mathbf{Y}+\mathbf{X}-\mathbf{s} \pm \mathbf{Z})$

Integrating over $\mathbf{z}$ we obtain the momentum conservation delta function $(2 \pi)^{3} \delta\left(\mathbf{Q}-\mathbf{p}_{0}+\mathbf{p}-\mathbf{p}^{\prime}\right)$ and integrating over $t$ the energy conserving delta function

$2 \pi \delta\left(E-\frac{\mathbf{p}_{0}^{2}}{2 \mu}-\frac{\mathbf{p}^{\prime 2}}{2 m}+\frac{\mathbf{p}^{2}}{2 m}-V_{M F}(\mathbf{Y})\right)$.

Now using the Gaussian parametrization (2.13) for the effective two body interaction $u$ and changing to the variables $\xi=\mathbf{s}-\mathbf{s}^{\prime}$ and $\xi^{\prime}=\frac{\mathbf{s}+\mathbf{s}^{\prime}}{2}$ one obtains finally (2.19) and (2.20).

\section{Appendix B. Momentum Integrations in $W(E, Y, Q)$}

The sequence of momentum integrations which appear in (2.19) and (2.20) is performed as follows.

The axis $q$ is the difference in the momenta of particle and hole, $\mathbf{q}=\mathbf{p}-\mathbf{p}^{\prime}$ and we let $\mathbf{P}=\left(\mathbf{p}+\mathbf{p}^{\prime}\right) / 2$.

In order to perform the momentum integrations it is convenient to take as reference axis for the qintegration the fixed conjugate momentum to $\mathbf{Y}, \mathbf{Q}$, and for the $\mathbf{P}=\left(\mathbf{P}_{z}, \mathbf{P}_{1}\right)$ integration the axis $\mathbf{q}$, alined along $P_{Z}$.

After integrating over $p_{0}$ in (2.19) we express the energy-conserving delta function in the new variables $\mathbf{q}$ and $\mathbf{P}$

$$
\begin{aligned}
& \delta\left(E-\frac{1}{2 \mu}(\mathbf{Q}+\mathbf{q})^{2}-\frac{(\mathbf{P}-\mathbf{q} / 2)^{2}}{2 m}+\frac{(\mathbf{P}+\mathbf{q} / 2)^{2}}{2 m}-V_{M F}(\mathbf{Y})\right) \\
& =\frac{\mu}{Q q} \delta\left(\cos \theta+\frac{1}{2 Q q}\left(Q^{2}+q^{2}-2 \mu\left(E-V_{M F}(\mathbf{Y})\right)\right.\right. \\
& \left.\left.\quad-\frac{2 \mu}{m} P_{z} q\right)\right) .
\end{aligned}
$$

An integration over the $\cos \theta$ in the delta-function leads to the condition

$\left|E+\frac{P_{z} q}{m}-\frac{Q^{2}+q^{2}}{2 \mu}-V_{M F}(\mathbf{Y})\right| \leqq \frac{Q q}{\mu}$.

In the following we restrict our analysis to the energyshell condition $E=\frac{Q^{2}}{2 \mu}+V_{M F}(\mathbf{Y})$, which, inserted in (B.2) constrains the $P_{Z}$-momentum region to

$-\frac{m}{\mu}\left(Q+\frac{q}{2}\right)<P_{z}<\frac{m}{\mu}\left(Q-\frac{q}{2}\right)$.

We get therefore

$$
\begin{aligned}
& W(E(\mathbf{Q}), \mathbf{Y})=2\left(\frac{\pi r_{0}^{2}}{\sqrt{2}}\right)^{3 / 2} \frac{u_{0}^{2}}{(2 \pi)^{4}} \frac{\mu}{Q} \int_{0}^{\infty} \mathrm{d} q q \mathrm{e}^{-\frac{r_{0}^{2}}{2} q^{2}} \\
& \cdot \int \mathrm{d} \mathbf{X} \mathscr{F}[\mathbf{Y}+\mathbf{X},-\mathbf{q}] \int_{-\frac{m}{\mu}\left(Q+\frac{q}{2}\right)} \mathrm{d} P_{z} \int \mathrm{d} \mathbf{P}_{\perp} \\
& \cdot \Theta\left(\lambda-\frac{1}{2 m}\left(\mathbf{P}_{\perp}^{2}+P_{z}^{2}+\frac{q^{2}}{4}+P_{z} q\right)-V_{0}(\mathbf{X})\right) . \\
& \left.\Theta\left(\frac{1}{2 m}\left(\mathbf{P}_{\perp}^{2}+P_{z}^{2}+\frac{q^{2}}{4}-P_{z} q\right)+V_{0}(\mathbf{X})-\lambda\right)\right)
\end{aligned}
$$


First we do the $P_{\perp}$-integration and obtain from the product of the $\Theta$-functions, which we denote by $\Theta_{1}$ and $\Theta_{2}$

$\int \mathrm{d} \mathbf{P}_{\perp} \Theta_{1} \Theta_{2}=2 \pi P_{z} q \quad$ for $P_{\perp \mathrm{MIN}}^{2}>0 ; \quad P_{\perp \mathrm{MAX}}^{2}>0$

or

$\int \mathrm{d} \mathbf{P}_{\perp} \Theta_{1} \Theta_{2}=\pi\left(P_{F}^{2}(\mathbf{X})-\left(P_{z}-\frac{q}{2}\right)^{2}\right)$

if $P_{\perp \mathrm{MIN}}^{2}<0 ; P_{\perp \mathrm{MAX}}^{2}>0$.

Here $P_{\perp \text { MIN }}^{2}$ and $P_{\perp \text { MAX }}^{2}$ denote the boundaries of the $P_{\perp}^{2}$-integration which stem from the product $\Theta_{1} \Theta_{2}$,

$P_{\perp \mathrm{MIN}}^{2}=P_{F}^{2}(\mathbf{X})-\left(P_{z}+\frac{q}{2}\right)^{2}$

$P_{\perp \mathrm{MAX}}^{2}=P_{F}^{2}(\mathbf{X})-\left(P_{z}-\frac{q}{2}\right)^{2}$

and

$P_{F}^{2}(\mathbf{X})=2 m\left(\lambda-V_{0}(\mathbf{X})\right)$.

Now we perform the $P_{z}$-integration. We introduce the following notations

$$
\begin{aligned}
& \Gamma=\frac{1}{2 \frac{\mu}{m}+1} \\
& \Delta=\frac{2}{1-\frac{m}{\mu}}\left(P_{F}(X)-\frac{m}{\mu} Q\right) \\
& \Delta_{1}=\frac{2}{1+\frac{m}{\mu}}\left(\frac{m}{\mu} Q-P_{F}(\mathbf{X})\right) \\
& \Delta_{2}=\frac{2}{1+\frac{m}{\mu}}\left(\frac{m}{\mu} Q+P_{F}(\mathbf{X})\right) \\
& f\left(P_{z}\right)=\left[P_{F}^{2}(\mathbf{X})-\left(P_{Z}-\frac{q}{2}\right)^{2}\right] \\
& g\left(P_{z}\right)=2 P_{z} q .
\end{aligned}
$$

The result of the $P_{Z}$-integration is summarized in the following equations (B.15) and (B.16).

$$
\int \mathrm{d} P_{z} g\left(P_{z}\right)=D 1=q\left(P_{F}(X)-\frac{q}{2}\right)^{2}
$$

$$
\text { for }\left\{\begin{array}{l}
\Delta \leqq q \leqq 2 P_{F}(\mathbf{X}) \\
\text { if } \frac{m}{\mu} Q \leqq P_{F}(\mathbf{X}) \leqq Q \\
\text { or } \\
0 \leqq q \leqq 2 P_{F}(\mathbf{X}) \\
\text { if } 0 \leqq P_{F}(\mathbf{X}) \leqq \frac{m}{\mu} Q
\end{array}\right.
$$

$\int \mathrm{d} P_{z} g\left(P_{z}\right)=D 2=\left(\frac{m}{\mu}\right)^{2} q\left(Q-\frac{q}{2}\right)^{2}$

for $\left\{\begin{array}{l}0 \leqq q \leqq 2 Q \\ \text { if } P_{F}(\mathbf{X}) \geqq Q \\ \text { or } \\ 0 \leqq q \leqq \Delta \\ \text { if } \frac{m}{\mu} Q \leqq P_{F}(\mathbf{X}) \leqq Q\end{array}\right.$

$\int \mathrm{d} P_{z} f\left(P_{z}\right)=D 3=P_{F}(\mathbf{X}) q^{2}-\frac{1}{3} q^{3}$

for $\left\{\begin{array}{l}0<q<2 P_{F}(\mathbf{X}) \\ \text { if } P_{F}(\mathbf{X}) \leqq \Gamma Q \\ \text { or } \\ 0<q<\Delta_{1} \\ \text { if } \Gamma Q \leqq P_{F}(\mathbf{X}) \leqq \frac{m}{\mu} Q\end{array}\right.$

$\int \mathrm{d} P_{z} f\left(P_{z}\right)=D 4=\frac{4}{3} P_{F}^{3}(\mathbf{X})$

for $\left\{\begin{array}{l}2 P_{F}(\mathbf{X})<q<\Delta_{1} \\ \text { if } P_{F}(\mathbf{X}) \leqq \Gamma Q\end{array}\right.$

$$
\begin{aligned}
\int \mathrm{d} P_{z} f\left(P_{z}\right) & =D 5=\left\{P_{F}^{2}(\mathbf{X})\left[\frac{m}{\mu}\left(Q-\frac{q}{2}\right)-P_{F}(\mathbf{X})+\frac{q}{2}\right]\right. \\
& \left.-\frac{1}{3}\left[\left(\frac{m}{\mu}\left(Q-\frac{q}{2}\right)-\frac{q}{2}\right]^{3}-\left(P_{F}(\mathbf{X})-q\right)^{3}\right]\right\}
\end{aligned}
$$

for $\left\{\begin{array}{l}\Delta \leqq q \leqq 2 P_{F}(\mathbf{X}) \\ \text { if } Q \geqq P_{F}(\mathbf{X}) \geqq \frac{m}{\mu} Q \\ \text { or } \\ \Delta_{1} \leqq q \leqq 2 P_{F}(\mathbf{X}) \\ \text { if } Q \Gamma \leqq P_{F}(\mathbf{X}) \leqq \frac{m}{\mu} Q\end{array}\right.$ 


$$
\begin{aligned}
& \int \mathrm{d} P_{z} f\left(P_{z}\right)=D 6=P_{F}^{2}(\mathbf{X})\left(\frac{m}{\mu}\left(Q-\frac{q}{2}\right)-\frac{q}{2}\right) \\
& \left.-\frac{1}{3}\left(\frac{m}{\mu}\left(Q-\frac{q}{2}\right)-\frac{q}{2}\right)^{3}\right)+\frac{2}{3} P_{F}^{3}(\mathbf{X}) \\
& \text { for }\left\{\begin{array}{l}
2 P_{F}(\mathbf{X}) \leqq q \leqq \Delta_{2} \\
\text { if } \frac{m}{\mu} Q \geqq P_{F}(\mathbf{X}) \geqq \Gamma Q \\
\text { or } \\
\Delta_{1} \leqq q \leqq \Delta_{2} \\
\text { if } P_{F}(\mathbf{X})<\Gamma Q \\
\text { or } \\
2 P_{F}(\mathbf{X}) \leqq q \leqq \Delta_{2} \\
\text { if } \frac{m}{\mu} Q \leqq P_{F}(\mathbf{X}) \leqq Q
\end{array}\right.
\end{aligned}
$$

The $P_{z}$-integration has generated a lot of step-functions for the $q$-integrations.

Let the function $F$ contain the $q$-dependence of $W(E(Q), Y)$ which is not included in $\int \mathrm{d} P_{z} g\left(P_{z}\right)$ and $\int \mathrm{d} P_{z} f\left(P_{z}\right)$. The explicit form of $F$ is given in (B.18) below. We end up with the following regions in momentum space. In the equation (B.17) below the quantities appearing in curly brackets are abbreviated in the main text as $I(q, X)$, see (3.6) and (3.8).

$$
\begin{aligned}
& \text { Region } I: 0 \leqq P_{F}(X) \leqq \Gamma Q \\
& \int d q \Theta(q) F\left\{(D 1+D 3) \Theta\left(2 P_{F}(X)-q\right)\right. \\
& \quad+D 4 \cdot \Theta\left(q-2 P_{F}(X)\right) \Theta\left(\Delta_{1}-q\right) \\
& \left.\quad+D 6 \cdot \Theta\left(q-\Delta_{1}\right) \Theta\left(\Delta_{2}-q\right)\right\} \\
& \text { Region II: } \Gamma Q \leqq P_{F}(X) \leqq \frac{m}{\mu} Q \\
& \int \mathrm{d} q \Theta(q) F\left\{(D 1+D 3) \Theta\left(\Delta_{1}-q\right)\right. \\
& \quad+(D 1+D 5) \Theta\left(q-\Delta_{1}\right) \Theta\left(2 P_{F}(X)-q\right) \\
& \left.\quad+D 6 \Theta\left(2 P_{F}(X)-q\right) \Theta\left(A_{2}-q\right)\right\} \\
& \operatorname{Region} I I I: \frac{m}{\mu} Q \leqq P_{F}(X) \leqq Q \\
& \int \mathrm{d} q \Theta(q) F\left\{D 1 \cdot \Theta(q-A) \Theta\left(2 P_{F}(X)-q\right)\right. \\
& \quad+D 2 \cdot \Theta(\Delta-q)+D 5 \cdot \Theta(q-A) \cdot \Theta\left(2 P_{F}(X)-q\right) \\
& \left.\quad+D 6 \cdot \Theta\left(q-2 P_{F}(X)\right) \Theta\left(\Delta_{2}-q\right)\right\}
\end{aligned}
$$

Region $I V: P_{F}(X) \geqq Q$

$$
\int \mathrm{d} q \Theta(q) F\{D 2 \cdot \Theta(2 Q-q)\}
$$

where $D 1$ to $D 6$ are defined in (B.15) and (B.16).
The function $F$ which occurs in (B.17) assumes different forms depending on whether the single-particle density of the inert ion is a Gaussian or a symmetrized Woods-Saxon.

In the case of a Gaussian we obtain

$$
F=q \mathrm{e}^{-\frac{q^{2}}{2}\left(r_{0}^{2}+a^{2}\right)} \text {. }
$$

For Woods-Saxons with LMA it is

$$
F=\mathrm{e}^{-\frac{q^{2}}{2} r_{0}^{2}} \frac{R \cos (q R)-\frac{a \pi \sin (q R) \cosh (a \pi q)}{\sinh (a \pi q)}}{q \sinh ^{2}(a \pi q)}
$$

\section{Appendix C}

Here we sow that at large insident energy per particle, $E / A \gtrsim \lambda$, the imaginary part of the optical potential, $W(E(Q), Y)$, is inversely proportional to the momentum $Q$.

A relevant quantity which occurs in the momentum integrations is the Fermi momentum

$$
\bar{P}_{F}=\frac{P_{F}(X)}{\sqrt{m \lambda}}=\left[2\left(1-\frac{V_{0}(X)}{\lambda}\right)\right]^{1 / 2}
$$

in units of $\sqrt{m \lambda}$, whereby we set $\lambda=47 \mathrm{MeV}$ (for $A$ $=64$ ) and $\lambda=42 \mathrm{MeV}$ (for $A=12$ ). All barred quantities below are also in these units. The Fermi momentum is comprised between the values $0 \leqq \bar{P}_{F} \leqq \sqrt{2}$. We obtain the following results.

(i) $\bar{P}_{F}=0$. In this case we expect $W(E(Q), Y)$ to be identically zero, independently of the value of $Q$, since $\bar{P}_{F}=0$ corresponds to the classical turning point of the nuclear potentials. This is easily verified.

(ii) $0 \leqq \bar{P}_{F} \leqq \sqrt{2}$. The integration limits $\Delta, \Delta_{1}, \Delta_{2}$ depend explicitly on the value of the reduced mass $\mu$, see (B.10-B.12). In order to get a mass-independent estimate, we write $\bar{Q} \geqq A$. The lowest boundary of $\bar{Q}$, $A$, is obtained by setting $E / \lambda=A$ and $V_{M F}=0$ in $\bar{Q}=\sqrt{\frac{2 \mu}{m \lambda}\left(E-V_{M F}\right)}$. Only region I (B.17a) with $0 \leqq \bar{P}_{F} \leqq \Gamma \bar{Q}$ and region II (B.17b) with $\Gamma \bar{Q} \leqq \bar{P}_{F} \leqq \frac{m}{\mu} \bar{Q}$ contribute to the momentum integrations. We obtain

$\Gamma \bar{Q} \gtrsim 1 ; \quad \frac{m}{\mu} \bar{Q} \gtrsim 2 ; \quad \bar{\Delta}<0 ;$

$2(2-\sqrt{2}) \lesssim \bar{J}_{1} \lesssim 4 ; \quad 2(2+\sqrt{2}) \lesssim \bar{\Delta}_{2} \lesssim 4$. 
The $Q$-dependence enters explicitly in the evaluation of $D 5$ and $D 6$ and in the boundaries of the $q$-integration, in $D 3$ and $D 4$. The $q$-integration decreases at least as $L=\exp \left(-\frac{q^{2} r_{0}^{2}}{2}\right)$. The largest value of $L$ in $D 5$ and $D 6$ is obtained at the smallest $q$-boundary, $\bar{A}_{1}$, and is small compared to the values $L$ assumes in $D 1$ and $D 3$ for $0<\bar{q}<\bar{\Delta}_{1}$. So we neglect $D 5$ and $D 6$, compared to $D 1$ and $D 3, D 1$ is independent of $Q$. Finally the $q$-integrations in $D 3$ and $D 4$ do not change with $Q$, which appears in the upper boundary $\bar{\Delta}_{1}$, because $L$ is then negligibly small. Therefore the only substantial $Q$-dependence appears in the denominator of $W(E(Q), Y)$, which is linear in $\bar{Q}$.

\section{References}

1. Feshbach, H.: Ann. Phys. 5, 357 (1958); 19, 287 (1962)

2. Vinh Mau, N.: Nucl. Phys. A457, 189 (1986)

3. Hasse, R.W., Schuck, P.: Nucl. Phys. A445, 205 (1985)

4. Buenerd, M. et al.: Nucl. Phys. A 424, 313 (1984)

5. Ghosh, G., Hasse, R.W., Schuck, P., Winter, J.; Phys. Rev. Lett. 50, 1250 (1983);

Blin, A.H., Hiller, B., Hasse, R.W., Schuck, P.: J. Phys. (Paris) 45, C6-231 (1984)

Blin, A.H., Hasse, R.W., Hiller, B., Schuck, P., Yannouleas, C.: Nucl. Phys. A456, 109 (1986); GSI-85-56 Preprint

6. Hasse, R.W., Schuck, P.: Nucl. Phys. A438, 157 (1985); J. Phys. Paris 45, C6-45 (1984)

7. Blin, A.H., Hasse, R.W., Hiller, B., Schuck, P.: Phys. Lett. 161 B, $211(1985)$

8. Ring, P., Schuck, P.: The nuclear many body problem. Berlin, Heidelberg, New York: Springer 1980

9. Brack, M., Guet, C., Hakanson, H.B.: Phys. Rep. 123, 275 (1985)

10. Blin, A.H., Brack, M., Hiller, B., Werner, E.: Proceedings of XXI Winter School on Physics, Report IFJ No. 1344/ps, p. 346 7-13 April, 1986, Zakopane, Poland; Proceedings of the International Workshop on Gross Properties of Nuclei and Nuclear Excitations XV, ISSNO 720-8715, 12-17 April 1987 Hirschegg, Austria; Interantional Workshop on Semiclassical and Phase Space Approaches to the Dynamics of the Nucleus, 1620 March, Aussois, France (in print)
11. Quesada, J.M., Broglia, R.A., Bragin, V., Pollarolo, G.: Proceedings of the International Conference on Theoretical Approaches of Heavy Ion Reaction Mechanisms. Martinot, M. (ed.) Paris 1984

12. Pollarolo, G., Broglia, R.A., Winther, A.: Nucl. Phys. A406, 369 (1983)

13. Broglia, R.A., Pollarolo G., Winther, A.: Nucl. Phys. A361, 307 (1981)

14. Jeukenne, J.P., Lejeune, A., Mahaux, C.: Phys. Rep. C10, 1391 (1974); C15, 10 (1977); C16, 80 (1977)

15. Saloner, D.A., Toepffer, C.: Nucl. Phys. A283, 108 (1987)

16. Izumoto, T., Krewald, S., Faessier, A.: Nucl. Phys. A341, 319 (1980);

Faessler, A., Izumoto, T, Krewald, S., Sartor, R.: Nucl. Phys. A359, 509 (1981);

Sartor, R., Faessler, A., Khadkikar, S.B., Krewald, S.: Nucl. Phys. A 357, 471 (1981);

Izumoto, T., Krewald, S., Faessler, A.: Nucl. Phys. A357, 471 (1981)

17. Khadkikar, S.B., Rikus, L., Faessler, A.: Nucl. Phys. A369, 495 (1981)

18. Decharge, J., Gogny, D.: Phys. Rev. C21, 1568 (1980);

Gogny, D.: Proceedings of the International Conference of Nuclear Selfconsistent Fields, Trieste, 1975, Ripka, G., Porneuf, M. (eds.) Amsterdam; North Holland 1975; Gogny, D., Padjen, R.: Nucl. Phys. A 293, 365 (1977)

19. Schuck, P., Bengston, R., Durand, M., Kunz, J., Ramamurthy, S.: In: Lecture Notes in Physics. Araki, H., Ehlers, J., Hepp, K., Kippenhahn, J., Weidenmüller, H.A., Zittartz, J. (eds.) Vol. 158: Dynamics of nuclear fission and related collective phenomena. David, P., Mayer-Kuckuk, T., Woude, A. van der (eds.), p. 183. Berlin, Heidelberg, New York: Springer 1982

20. Satchler, G.R., Love, W.G.: Phys. Rep. 55, 183 (1979)

21. Feshbach, H., Hüfner, J.: Ann. Phys. 56, 268 (1970);

Feshbach, H., Gal, A., Hüfner, J.: Ann. Phys. 66, 20 (1971)

22. Hasse, R.W.: Ann. Phys. 80, 118 (1973)

23. Grammaticos, B.: Ann. Phys. 126, 450 (1980)

24. Glauber, R.J.: In: Boulder lectures in theoretical physics. Vol. I. New York: Interscience 1959; Glauber, R.J., Mathiae, G.: Nucl. Phys. B 21, 135 (1970)

24. Satta, L. et al.: Phys. Lett. 139 B, 263 (1984)

A.H. Blin, M. Brack, B. Hiller, E. Werner

Institut für Theoretische Physik

Universität Regensburg

Universitätsstrasse 31

D-8400 Regensburg

Federal Republic of Germany 